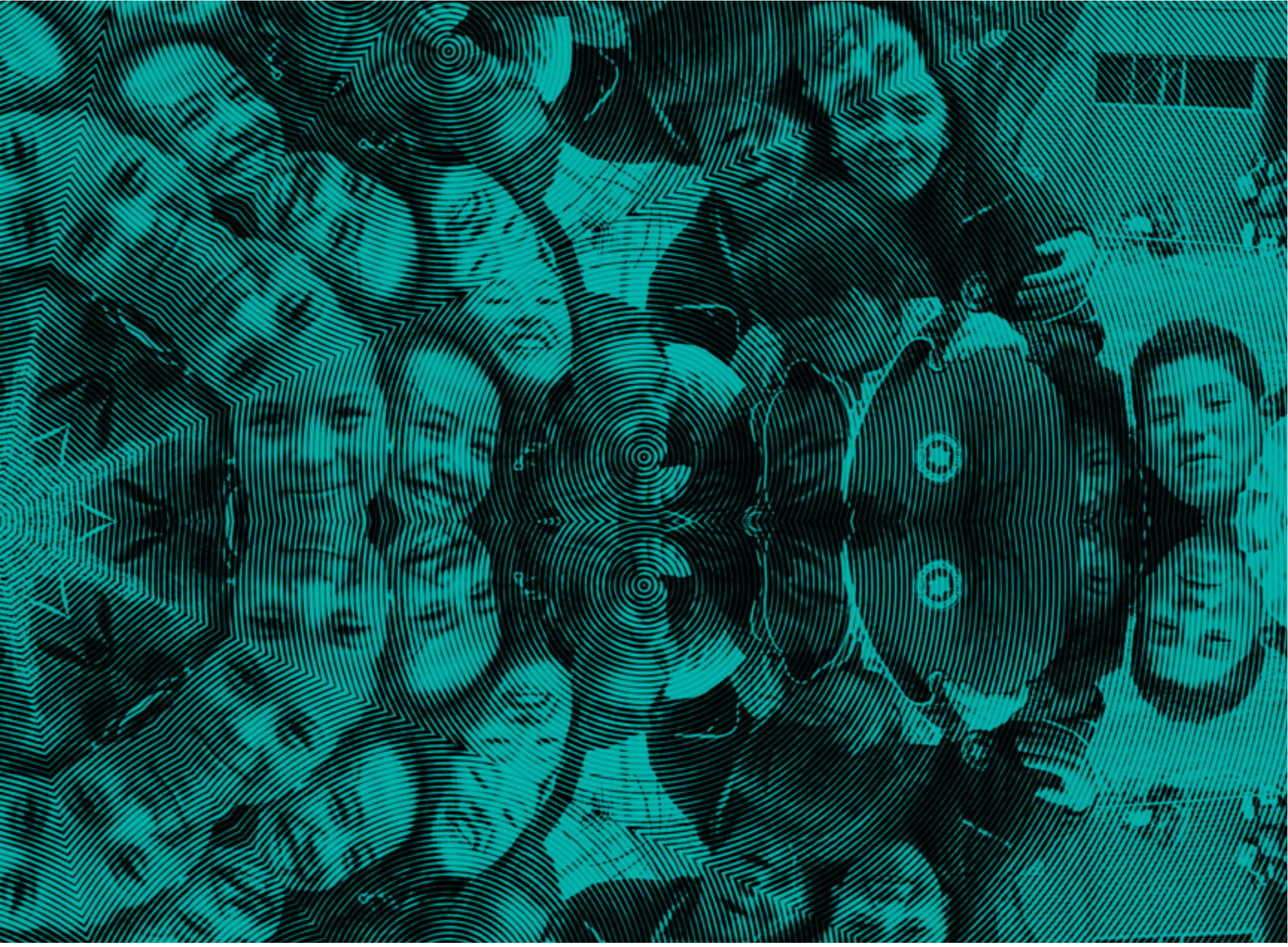

\title{
Relación del clima de aula y las emociones morales: culpa y empatía
}

The relation between classroom environment and moral emotion: empathy and guilt

Relação do ambientes educacionais e as emoções morais: culpa e empatía

Mary luz Esquivel Fajardo Martha Liliana Gutiérrez Herrera Angélica María Mercado Roa Yeimy Pachón Forero Gloria Patricia Ortega Roa 


\section{Mary luz Esquivel Fajardo 1 Martha Liliana Gutiérrez Herrera 2 Angélica María Mercado Roa 3 Yeimy Pachón Forero 4 Gloria Patricia Ortega Roa 5}

1. Correo electrónico: soyhumana@gmail.com

2. Correo electrónico: marthagu3@gmail.com

3. Correo electrónico: angiemr55@gmail.com

4. Correo electrónico: emylin.4@gmail.com

5. Correo electrónico: patriciaylucia@gmail.com

Agradecimientos a: Gloria Inés Rodríguez Directora de la tesis de grado. Doctora en Psicología.

Grupo de Trabajo. Docentes de la Secretaría de Educación del Distrito, Bogotá y Magister en Educación de la Pontificia Universidad Javeriana

Fecha de recepción: 18 de abril de 2016 / Fecha de aceptación: 16 de septiembre de 2016

\section{Resumen}

La investigación pretende determinar la relación entre el clima de aula y las emociones morales de empatía y culpa en estudiantes de grado sexto. El enfoque metodológico fue cuantitativo de tipo exploratorio, la muestra seleccionada estuvo compuesta por 189 estudiantes con un promedio de edad de 11 años. Se aplicó un cuestionario con escala tipo Likert previamente piloteado y validado. Los resultados de las correlaciones mostraron una asociación significativa positiva pero baja entre clima de aula y empatía, y una relación significativa positiva moderada entre clima de aula y culpa.

Palabras clave: Clima de aula, empatía, culpa, educación para la paz.

\section{Summary}

The investigation interest to determinate the relation between classroom environment and moral emotion related with empathy and guilt in students that are in sixth. The methodological investigation was focused by quantitative research based in exploratory, the selected sample has 189 students with an average age of 11 years old. An questionnaire using likert scale was applied with a previous pilot study and was validated. The correlation in research results was high, positive but with low incidence between classroom environment and empathy, while in the other side the same result shows a significant moderate positive relation between classroom environment and guilt.

\section{Keywords: Classroom} environment, Moral emotions, empathy, guilt, education for the peace.

\section{Resumo}

A investigação pretende determinar a relação entre ambientes educacionais e a presença de emoções morais: empatía e culpa em estudantes de grau sexto. $O$ enfoque metodológico da investigação foi quantitativo de tipo exploratorio, a mostra seleccionada esteve composta por 189 estudantes com uma média de idade de 11 ano. Aplicou-se um questionário com escala tipo Likert previamente piloteada e validado. Os resultados das correlações mostraram uma associação significativa, positiva mas baixa entre ambientes educacionais e empatía, enquanto este mesmo tipo de análise indicou uma relação significativa positiva moderada entre clima de sala e culpa.

Palavras chave: Ambientes educacionais, empatía, culpa, educação para a paz. 


\section{Introducción}

Una de las preocupaciones constantes de los maestros y maestras es el clima de aula y las relaciones que allí se gestan, dado que en el contexto escolar emergen múltiples maneras de solucionar conflictos a través de la agresión y la violencia, configurando relaciones destructivas y poco asertivas que en muchas ocasiones van en contravía de la moralidad, la justicia y el reconocimiento del otro; por esta razón es evidente que los procesos formativos deben incluir estrategias que permitan el desarrollo de habilidades interpersonales, promoviendo prácticas educativas en ambientes favorables y construyendo relaciones justas en el medio escolar; esto es importante si se piensa que una labor fundamental de la escuela es educar ciudadanos que convivan de forma pacífica y constructiva en sus comunidades.

Las investigaciones que se han llevado a cabo en este ámbito empiezan en los años 90s con Martínez (1996) y, posteriormente, Sánchez (2009) y Chaux (2012). Los autores convergen en que la importancia del clima de aula radica en la adecuada atmósfera de trabajo y la calidad de las relaciones entre pares (estudiantes) y docentes, así como en la institución de normas consensuadas. Elementos que pueden propiciar un desarrollo en las diferentes dimensiones del ser humano como lo social, cultural, moral e intelectual. En este orden de ideas, el interior de las aulas de clase es uno de los escenarios en los cuales se pueden propiciar relaciones de cuidado y bienestar o, por el contrario, generar ambientes de hostilidad y hasta agresión.

El estudio más reciente acerca del tema es la encuesta realizada por la Secretaría de Educación de Bogotá (2013), que ofrece un panorama sobre el clima escolar en las instituciones educativas, otorgando importancia a las interacciones, las relaciones y la cotidianidad creada en el aula, condicionada al tipo de práctica pedagógica. Los datos evidencian que en un 46\% los estudiantes toleraron burlas, interrupciones y conversaciones de otros en clase, lo cual genera múltiples distracciones, indicando que un $44 \%$ de los estudiantes percibían que no se podía dictar clase. Es posible que estos resultados sean el indicativo de cómo un deteriorado clima de aula afecta, tanto el proceso de aprendizaje de los estudiantes y las relaciones de convivencia, como las condiciones de los maestros y maestras que tienen que enfrentar ambientes de hostilidad y estrés.

Otro elemento importante para el desarrollo del clima de aula son las emociones morales, que han mostrado ser importantes para establecer relaciones prosociales y de cuidado entre los seres humanos; toda vez que lo moral tiene que ver con comprender la manera en que los seres humanos sienten, piensan, dicen y actúan con respecto a las normas sociales, y lo que es aceptable o no socialmente. El concepto de moral tiene varias categorías: el componente racional, donde el sujeto puede analizar y decidir sobre lo correcto o incorrecto dependiendo de las ideas, juicios y habilidades cognitivas; la interiorización de las normas, que depende de los contextos sociales donde se desenvuelve dicho sujeto; y el desarrollo emotivo, es decir, las diferentes emociones y sentimientos como base del comportamiento moral (Rodríguez, 2003).

Ahora bien, para esta investigación se tomó como referente las emociones morales que, de acuerdo a los planteamientos de Nussbaum (2008), nos permiten tener vínculos conscientes con los demás seres humanos a partir de procesos de reflexión intersubjetiva de lo que el sujeto valora y es moralmente aceptado, por ello son cognitivo-evaluadoras y fenómenos sociales que se construyen en un determinado contexto social. Las emociones morales han mostrado ser importantes para establecer relaciones prosociales y de cuidado entre los seres humanos. En estudios realizados por Hoffman (2002), y Bilbo, Urquijo, Barón, Rebollo y Sánchez (2009), se ha evidenciado que el desarrollo de la culpa y la empatía evitan el maltrato y promueven el bienestar de los demás.

Por un lado, la culpa es una emoción ligada a los valores de las personas y a la manera como se reacciona para reparar el daño y ser responsable con el otro (Rojas, 2009). Esta emoción tiene dos formas de asumirse: una positiva, relacionada con la toma de perspectiva y control de impulsos frente a conductas que generan daño individual o colectivo, buscando una acción que repare el daño causado; y otro negativo, que tiene que ver con la capacidad de sentir miedo, dolor y angustia, convirtiéndose en una carga tormentosa, lo que no permite trascender a tener un efecto reparador hacia el daño causado. Por lo tanto, la culpa manejada de manera positiva ayuda a construir relaciones sociales y tener conciencia del otro: "En la culpa, el agente acepta una responsabilidad por un comportamiento que viola estándares internalizados de conducta religiosa, moral o ética; se focaliza en condenar el hecho deseando enmendarlo o compensarlo o castigar al yo" (Rodríguez, 2003, p.26).

Por otro lado, la empatía es la acción de sentir algo que es más congruente con la situación de otro (Hoffman, 2002; Bilbo, Urquijo, Barón, Rebollo y Sánchez, 2009); se trata de una emoción congruente con el estado emocional del otro, es decir, colocarse en su lugar como una respuesta emocional que procede de comprender su estado o situación y es similar a lo que ese otro está sintiendo, 
es una variable importante en la conducta prosocial (Batson, citado por Fernández, 2008).

Frecuentemente en la cotidianidad escolar se ve que algunos estudiantes con problemas de agresividad muestran comportamientos poco empáticos; la aparición de la culpa en estos casos está asociada a la transgresión de normas y castigos impuestos por los adultos, más que como resultado de procesos dialógicos y de reflexivos sobre el bienestar colectivo y las relaciones de cuidado que se puedan dar en el aula. Así, un clima positivo en el aula toma gran relevancia a partir de dos componentes fundamentales: La estructura de la clase y el cuidado de las relaciones (Chaux, 2012); a los que se suma el componente normas como otro elemento importante.

La estructura de clase tiene que ver con la adecuación del ambiente, el mobiliario, los contenidos pedagógicos, materiales didácticos, y un conjunto de interrelaciones personales que implica una serie de acuerdos y normas que se desarrollan de manera conjunta entre docentes y estudiantes. Por su parte, las relaciones de cuidado son el conjunto de acciones que buscan atender las necesidades del otro por encima de las propias, e implican un desplazamiento motivacional en el marco de una comunicación de doble vía (Noddings, citado por Rivera, 2009, p. 29); así: “Cuidar y ser cuidado es una necesidad básica humana. El sentirse recibido por otras personas y el saberse capaz de motivar la respuesta de otros frente a una necesidad propia, son elementos centrales en la vida de cualquier individuo" (Chaux, Daza y Vega, 2008, p. 4).

Como docentes es importante considerar que en las relaciones de cuidado se deben tener en cuenta aspectos como: la preocupación mutua, la calidez, el afecto y la comunicación clara y abierta; los cuales llevan a relaciones de bienestar donde el docente, como orientador de la clase, debe garantizar que estos factores estén presentes en el clima de aula. La relación del profesor con sus estudiantes está tradicionalmente ligada a la idea de que los maestros son el factor crucial de la educación en el aula, pues a través de su práctica pedagógica pueden generar una atmósfera tranquila, ordenada y orientada al aprendizaje; al respecto:

La labor de enseñanza y el modelo de persona que el profesor proporciona a sus alumnos, contribuye [...] a la formación de la personalidad de los que serán, a su vez, los protagonistas del futuro. [...] Para ser eficaz como educador, el profesor puede y debe darse cuenta de lo que hace y de lo que puede hacer en su aula para crear un ambiente favorecedor de una buena autoestima de sus alumnos y de una convivencia que facilite esta labor (Voli, 2004, p. 78).

El docente es un agente facilitador y generador de emociones y relaciones adecuadas en el aula, porque las entiende como eje principal que impacta y es modelo para las acciones y relaciones

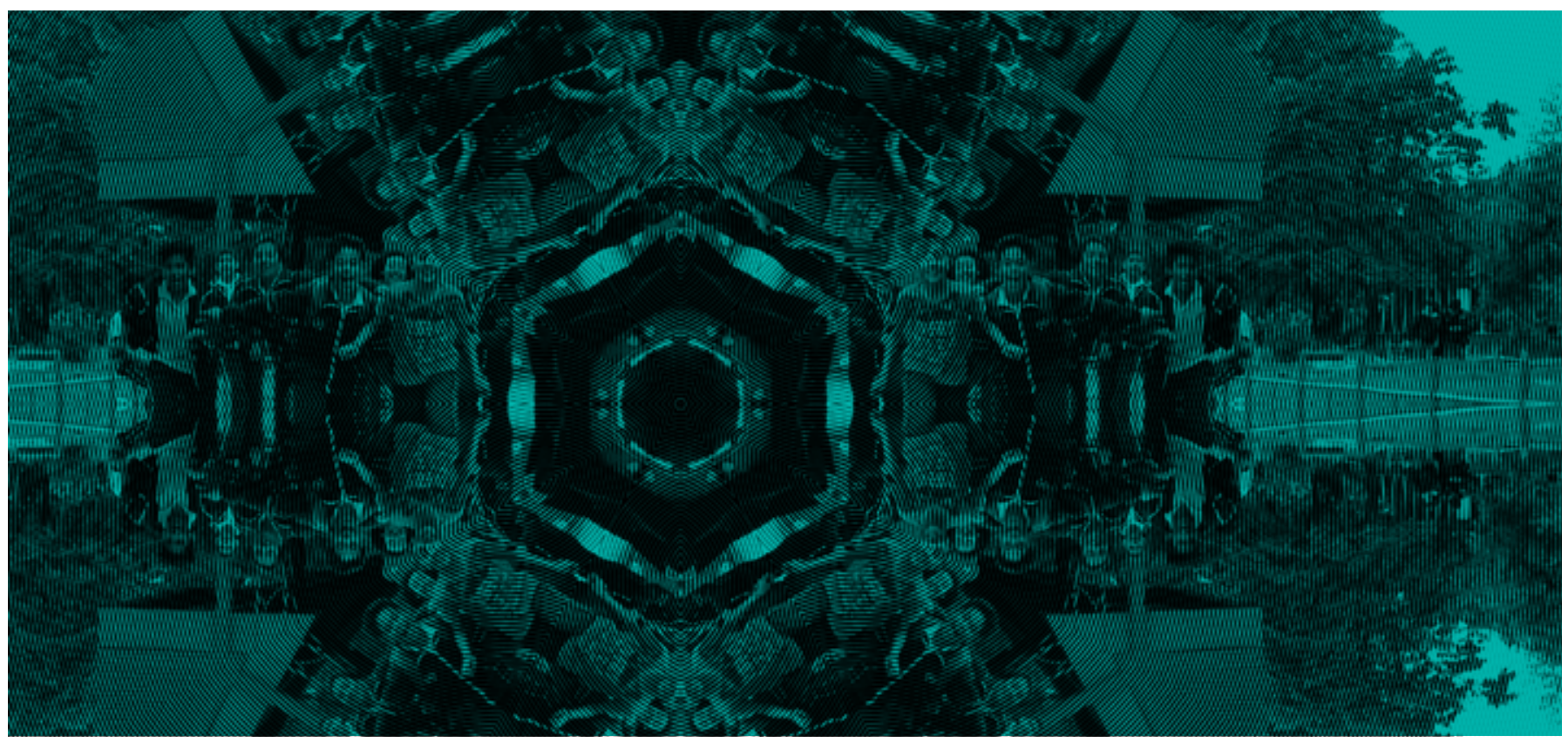


de sus estudiantes; el clima que se genere en el aula promoverá o no este tipo de relaciones entre ellos. Es pertinente pensar que los estilos autoritarios en los que muchos hemos crecido y que con frecuencia se reflejan también en la escuela, son contradictorios con la generación de ambientes de cuidado, ya que promueven relaciones de sumisión y miedo, en donde las necesidades de cada uno y el bienestar común pasan a segundo plano.

En el clima de aula también influyen las normas, la forma en que se establecen en el aula de clase hará que se entiendan como una imposición o como una forma de autorregulación indispensable para propiciar el bien común; en un clima de aula positivo, las normas, antes que imposiciones o parámetros para buscar utilidad, se constituyen en decisiones conscientes y reflexivas de los individuos a partir de preferencias y creencias (Bicchieri, 2006). Esto refuerza la idea de generar relaciones democráticas al interior del aula, que permitan la participación y la reflexión de los estudiantes alrededor de las normas necesarias para lograr el bienestar común. Las normas son inherentes a la convivencia en sociedad $\mathrm{y}$, al interior del aula, deben ser consensuadas, conocidas y aceptadas a partir de un diálogo de confianza entre docentes y estudiantes (Carrasco, et al, 2011).

Identificar la relación entre el clima de aula positivo y las emociones de empatía y culpa en estudiantes, permitirá ahondar en la comprensión del clima de aula y su relación con el fomento de las emociones morales; al tiempo que en el diseño de estrategias e implicaciones prácticas que mejoren el clima en el aula y promuevan la convivencia escolar pacífica.

\section{Muestra}

La investigación se desarrolló en la Localidad de San Cristóbal con cuatro instituciones distritales de la ciudad de Bogotá, trabajó una muestra de 189 estudiantes de los grados sextos de educación básica secundaria, 90 del género femenino y 99 del masculino, con edades comprendidas entre los 10 y los 14 años: 1 estudiante de 10 años, 75 de 11 años, 61 de 12 años, 40 de 13 años y 12 de 14 años. Para determinar el número de estudiantes de cada institución se usó un muestreo aleatorio simple, con confiabilidad del 95\% y error de estimación del 5\%.

\section{Procedimiento}

La muestra fue seleccionada por conveniencia, pues las investigadoras tienen acceso directo a los grupos en las instituciones donde laboran. En las cuatro entidades educativas se aplicó un cuestionario a la muestra seleccionada, y se elaboró un consentimiento informado donde los padres de familia daban el aval para que sus hijos lo presentaran. Para la aplicación del instrumento se procedió a crear un protocolo de instrucciones para las investigadoras, con el fin de mantener criterios similares en su aplicación en cada una de las instituciones.

\section{Instrumentos}

Se diseñó un cuestionario que incluyó una escala tipo Likert con las tres categorías de estudio: Clima de aula, culpa, y empatía. El resultado final del instrumento fue de 54 preguntas: cincuenta para contestar con la escala tipo Likert (siempre (5), casi siempre (4), algunas veces (3), casi nunca (2), nunca (1)), y cuatro para contestar con respuesta de Sí o No. Se realizó un análisis de confiabilidad o consistencia interna a partir del alfa Cronbach, donde se encontró una consistencia de 0,746 para la dimensión de clima de aula, en la dimensión de empatía fue de 0,771, y en la de culpa fue de 0,733 .

\section{Resultados}

Para responder a la pregunta ¿Existe relación entre el clima de aula positivo y la presencia de emociones de empatía y culpa en estudiantes de sexto grado de la ciudad de Bogotá?, en primer lugar se realizó un análisis de correlación entre las variables: clima de aula, culpa y empatía; debido a que todas las variables mostraron una distribución normal, se utilizó el coeficiente de correlación de Pearson. En la Tabla 1 pueden observarse los resultados. 
Tabla 1. Correlaciones entre las variables clima, empatía y culpa

\begin{tabular}{|l|l|r|r|r|}
\hline \multicolumn{5}{|c|}{ Correlaciones } \\
\hline \multirow{3}{*}{ Clima } & Correlación de Pearson & 1 &, $296^{\star \star}$ &, $572^{\star \star}$ \\
\cline { 2 - 5 } & Sig. (bilateral) & &, 000 &, 000 \\
\cline { 2 - 5 } & $\mathrm{N}$ & 165 & 163 & 162 \\
\hline \multirow{3}{*}{ Empatía } & Correlación de Pearson &, $296^{\star \star}$ & 1 &, $567^{\star \star}$ \\
\cline { 2 - 5 } & Sig. (bilateral) &, 000 & &, 000 \\
\cline { 2 - 5 } & $\mathrm{N}$ & 163 & 185 & 180 \\
\hline \multirow{3}{*}{ Culpa } & Correlación de Pearson &, $572^{\star \star}$ &, $567^{\star \star}$ & 1 \\
\cline { 2 - 5 } & Sig. (bilateral) &, 000 &, 000 & \\
\cline { 2 - 5 } & $\mathrm{N}$ & 162 & 180 & 184 \\
\hline \multirow{2}{*}{ ** La correlación es significativa al nivel 0,01 (bilateral). } \\
\hline
\end{tabular}

Según los análisis existe una correlación significativa positiva alta entre las variables clima de aula y culpa $(r=0.57, p=.00)$, mientras que las variables clima de aula y empatía presentan una correlación significativa y positiva, aunque baja $(\mathrm{r}=0,29, p=0.00)$.

En segundo lugar, se realizó un análisis de regresión lineal entre las variables, debido a que las correlaciones indicaron la existencia de relación entre clima de aula y culpa, y entre clima de aula y empatía; se realizaron algunos modelos de regresión lineal para determinar si las variables dependientes, culpa y empatía, eran explicadas por la independiente: clima de aula (Ver tabla 2)

Tabla 2. Resumen del modelo entre la variable independiente, clima de aula, con las variables dependientes culpa y empatía

\begin{tabular}{|l|r|r|r|r|}
\hline \multicolumn{1}{|c|}{ Modelo 1 } & R & R2 & R2 Corregido & $\begin{array}{c}\text { Error tip. De la } \\
\text { estimación }\end{array}$ \\
\hline Clima de aula - Culpa &, $572 a$ &, 328 &, 323 &, 54154 \\
\hline Clima de aula - Empatía &, $296 a$ &, 088 &, 082 &, 60242 \\
\hline
\end{tabular}

Debido a que el clima de aula explica la presencia de culpa en el aula de clase en $32,8 \%$, y de igual forma explica la presencia de empatía en un $8,8 \%$, se decidió hacer un análisis de regresión lineal múltiple entre las variables dependientes y las variables independientes; los componentes del clima incluidos en este estudio fueron: estructura de clase, relaciones de cuidado y normas. 
que se pueden observar en el aula con las diferentes relaciones que se gestan allí, motivadas por los diferentes estilos manejados por los docentes.

\section{Clima de aula: su} relación con empatía, relaciones de cuidado y estructura de clase

En nuestra investigación la relación directa entre clima de aula y empatía es positiva, la escuela es uno de los escenarios para que los jóvenes socialicen y adquieran capacidad empática. En esta obtención las prácticas pedagógi-

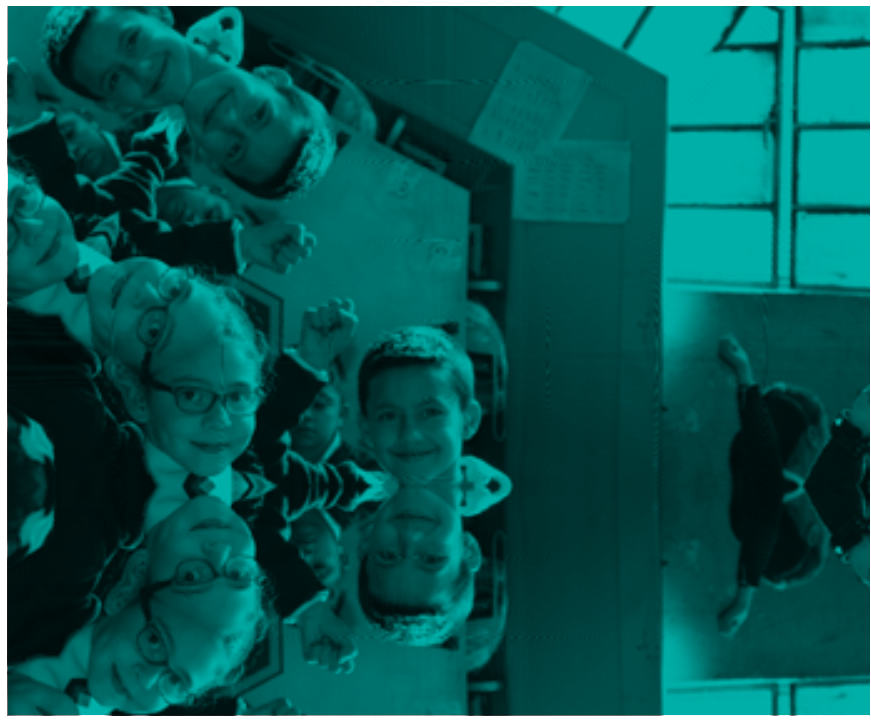

prevalencia del estilo negligente, debido a que hay una despreocupación por las normas y el afecto, y por lo tanto una desconexión entre docente y estudiante.

Los resultados de esta investigación, frente a la relación entre clima de aula y empatía, sugieren un desafío para la institución escolar; es importante incluir a la familia y al estudiante en procesos pedagógicos que estimulen la adquisición de esta emoción, especialmente en la escuela primaria. Lo anterior se sustenta en autores como Cepeda (2004), quien afirma que la empatía se adquiere cuando el niño tiecas y el clima de aula, promovido por el docente, son los elementos fundamentales para que, desde la cotidianidad escolar y las experiencias vividas, se desarrolle esta capacidad. Huegun (2009) reafirma nuestro estudio cuando señala que: "La generación de un clima relacional se sustenta en el respeto y la aceptación de las experiencias que comunican los jóvenes, y se plasma a través de las respuestas interaccionales y verbales concretas que ofrece el educador" (p, 16).

Las relaciones de cuidado dentro del aula permitirán afianzar la capacidad empática, pues los estudiantes se sentirán en un ambiente de confianza, de respeto entre profesores y pares, y evitarán agresiones, promoviendo un clima de aula positivo. Estudios como el de Eisenberg (2000) señalan que la sensibilidad empática puede favorecer el altruismo y reprimir la agresión. Otra categoría dentro del clima de aula es la estructura, que para esta población es negativa, situación que podría indicar que las estrategias metodológicas usadas por los docentes pueden no promover trabajos cooperativos, así como las respuestas empáticas ante situaciones de aislamiento de compañeros de clase, o de interacción con compañeros diferentes al grupo de pares habitual.

En el estudio los estudiantes manifestaron que, en situaciones que ameritan una intervención para dirimir posibles interrupciones de clase, la mayoría de docentes hace caso omiso y no manifiesta respuestas empáticas ante situaciones de malestar y maltrato en el grupo. Teniendo en cuenta la clasificación del estilo docente de Chaux (2012), los resultados del estudio permiten concluir que la práctica educativa de los docentes se puede caracterizar por la ne una serie de condiciones, en su entorno familiar y escolar, que le permitan experimentar y expresar sus emociones y entender las de los demás, saliendo del egocentrismo.

Estudios como el de Moreno, Estevez, Murqui y Musito (2009) sugieren que existe un vínculo entre el clima familiar y el comportamiento empático del estudiante en su contexto escolar; si al interior de la familia se presentan conflictos, falta de comunicación y cohesión afectiva, éste tendrá dificultades para relacionarse con sus pares y profesores en la toma de perspectiva y en la capacidad de comprender los sentimientos del otro. Nuestros resultados confirman dicha idea, toda vez que analizando las relaciones de cuidado y el clima de aula, con respecto a la empatía, se observó una relación positiva. Lo anterior debe ser considerado cuidadosamente, pues aunque los resultados obtenidos son estadísticamente significativos, las correlaciones entre las variables son bajas y moderadas para la muestra.

\section{Clima de aula, su relación con la culpa, relaciones de cuidado y normas}

La relación culpa y clima de aula presenta una asociación significativa positiva. Para nuestra investigación la culpa fue abordada desde la perspectiva de una emoción asociada con una respuesta empática hacia otros, que ofrece la posibilidad de poner en perspectiva los acontecimientos. En esta medida, se puede considerar como una emoción guardián de nuestra conducta y nos sirve de guía, funciona como un controlador de nuestros impulsos. 
La culpa es un conjunto emocional con diversos componentes, no únicamente implica las cuestiones morales; sus alcances están relacionados con normas y metas que tienen su origen en las preocupaciones intrapersonales. Así pues, en nuestro estudio se evidenció que el clima de aula tiene una mayor relación con la culpa, posiblemente porque gran mayoría los preadolescentes han interiorizado las normas en sus diferentes espacios sociales, incluido el del aula: la culpa en los adolescentes se presenta cuando se incumplen leyes sociales, obviando las cuestiones tradicionales que causa (mentir, hacer trampa).

Las relaciones de cuidado con respecto a la culpa y el establecimiento de normas fueron significativas; aunque la culpa es una emoción auto-evaluativa, se afianza a través de procesos de socialización, configurándose en una práctica de grupo, lo cual es de gran importancia en el aula de clases, dado que allí sí se construyen relaciones más cercanas, haciendo que ésta sea comprendida más allá de la perspectiva de una moral del deber ser (Rojas, 2009).

La norma, como suceso de respuesta a otros por la valoración del bien común, y no desde su perspectiva jurídica-legal, que implica seguir la sanción y el castigo, se refiere a un criterio ético de participación, reflexión y reparación. En este sentido, a través ella el maestro promueve procesos de miedo al castigo o enfocados en la culpa reparadora (Bustelo, 1998).

Para finalizar, es indudable la necesidad de afianzar la educación moral dentro del aula para fortalecer las relaciones de convivencia entre pares y entre estudiantes y docentes. Uno de los aportes a destacar en esta investigación fue la exploración de un nuevo campo dentro del contexto educativo, poco abordado en nuestro país, que permitirá generar nuevas investigaciones. A su vez, fue posible construir un instrumento base para evaluar la relación entre el clima de aula y las emociones morales. Aunque debemos tomar con precaución los resultados obtenidos debido a que la muestra es pequeña, son útiles como referencia para reflexionar sobre cómo se percibe la relación entre clima de aula y las emociones morales, de tal manera que mejore la práctica docente. 


\section{Bibliografía}

Abarca Castillo, M. (2003). La Educación Emocional en la educación primaria: currículo y práctica. Tesis de Doctorado no publicada. Universidad de Barcelona

Arias y Delgado. (2009). Educación en valores y ciudadania desde una perspectiva cotidiana. Bogotá IDIE, pp. 44-62.

Barreda Gómez, S. (2012). El docente como gestor del clima de aula. Factores a tener en cuenta. Tesis de Maestría no publicada. Formación del profesorado Educación secundaria. Universidad de Cantabria. España.

Berkowitz, W. (1995). Educar la persona moral en su totalidad. Revista Iberoamericana de Educación (8), pp. 73-101.

Bermúdez, B. (2011). La construcción de la confianza en la relación estudiante-docente en la Facultad de Administración de la UNAL de Colombia. Tesis de Maestría no publicada. Universidad Nacional de Colombia.

Bicchieri, C. (2006). La gramática de la sociedad. La naturaleza y la dinámica de las normas sociales. Cambridge: Cambridge UniversityPress.

Bilbo, I., Urquijo, P., Barón, M., Rebollo, M., y Sánchez, F. (2009). Emociones morales y conducta en niños y niñas. Revista de psicología y educación, Edupsykhé, Vol. 8, No. 1, p. 3.

Bonilla, E. (1997). Más allá del dilema de los métodos. La investigación en Ciencias Sociales. Barcelona: Uniandes.

Bustelo, E. (1998). Todos entran: propuesta para sociedades incluyentes. Bogotá: Santillana.

Carrasco, N., Orozco, M., Pino, S., y Vargas, V. (2011) ¿Qué relación existe entre el clima emocional de aula y el rendimiento escolar de niños y niñas de $2^{\circ}$ año de básico de una escuela Municipal de Cerro Navia. Tesis de Maestría no publicada. Universidad Academia de Cristianismo Humano. Santiago.

Casassus, J. (2003). La escuela y la (des) igualdad. Santiago: Colección Escafandra.

Cepeda, M. J. (2004). Ciudadanía y Estado Social de Derecho. Trabajo presentado en el Foro Educativo Nacional de Competencias Ciudadanas.
Bogotá, octubre 25. Obtenido desde http://www.mineducacion.gov. co/1621/articles-116042_archivo_pdf4.pdf

Cepeda, A. (2004). La relación inversa entre empatía y agresión. Bogotá: Universidad de los Andes.

Cortina, A. (1996). Ética minima.: Introducción a la filosofía práctica. Madrid: Tecnos.

Chaux, E., Daza, B., Vega, L. (2008). Las relaciones de cuidado en el aula y la institución educativa. Obtenido desde: http://www.colombiaaprende.edu. co/html/mediateca/1607/articles-168209_archivo.pdf

Chaux, E. (2012). Educación, convivencia y agresión escolar. Bogotá: Taurus.

Daza, Bertha. (2009). Educación en valores y ciudadanía desde una perspectiva cotidiana. Bogotá: IDIE, pp. 32-41.

De Zubiría, J. (2006). La violencia en los colegios de Bogotá. Obtenido desde http://www.institutomerani.edu.co/publicaciones/articulos/2009/ La_violencia_en_los_colegios_de_Bogot $\%$ C3\%A1.pdf.

Echeburúa, Corral y Amor. (2001). Estrategias de afrontamiento ante los sentimientos de culpa. Revista Análisis y modificación de conducta, Vol. 27, No. 116.

Einsenberg, N. (1976). Conducta prosocial. Obtenido el 6 de septiembre de 2014, desde http://reeduca.com/desarrollomoral-altruismoeisenb.aspx

Einsenberg, N. (2000). Emotion, regulation and moral development. Annu. Rev. Psychol, 51, pp. 665-697.

Fernández, P. (2008-diciembre). Empatía: Medidas, teorías y aplicaciones en revisión. Anales de psicología, Vol. 24, No. 2. Universidad Autónoma de Madrid.

García, Haro, J. (2015). Tres concepciones de la culpa escolar como factor de calidad. Madrid: Narcea ediciones.

Gutiérrez, M. (2008). Batería de instrumentos para la evaluación del clima escolar en escuelas primarias. México: Instituto Nacional para la Evaluación 
de la educación INEE. Obtenido desde http://blog.educastur.es/ autoevaluacion/files/2008/02/bateria-de-instrumentos-para-la-evaluacion-del-clima-escolar.pdf

Hansberg, O. E. (1996). De las emociones morales. México: Universidad Autónoma de México.

Hoffman, M. L. (2002). Desarrollo moral y empatía: implicaciones para la atención y la justicia. Cornellà del Lobregat: Idea Books.

Huegun, A. (2009, octubre-diciembre). La importancia de la empatía en el trabajo del educador para unas relaciones saludables. A tu salud. Revista de la Universidad Vasca (67-68), pp.15-19.

Laorden Gutiérrez, C. (2005). Educar emociones: un instrumento para trabajar el sentimiento de culpa. Revista Pulso, No. 28, pp. 125-138.

Martínez, M. (1996). El clima de la clase. Barcelona: Wolters Kulwer.

Monje, C. (2011). Metodología de la investigación cuantitativa y cualitativa. Neiva: Universidad Sur Colombiana. Colombia.

Moreno, D., Estevez, E., Murqui, S., y Musito, G. (s.f.). Relación entre el clima familiar y el clima escolar: el rol de la empatía y la actitud hacia la autoridad en la conducta de violencia en la adolescencia. International Journal Psychology and Psychological Therapy, 9(1), pp. 125-133.

Nussbaum, M. (2006). El ocultamiento de lo bumano. Buenos Aires: Katz.

Nussbaum, M. (2008). Paisajes del pensamiento. La inteligencia de las emociones. Barcelona: Paidós.

Olson, C.O., y Wyett, J. L. (2000). Teachers need affective competencies. Education, Vol. 120. Obtenido desde http://findarticles.com/p/articles/mi_qa3673/ is_200007/ai_n8902469

Ortega y Del Rey. (2007). Violencia escolar: claves para comprenderla y afrontarla. Revista Departamento de Psicología Evolutiva y de la Educación. 10, pp. 77-89. Universidad de Córdoba.

Pérez, L. (2012). Normas de aula y proactividad. Hacia la disciplina positiva. Navarra: Gobierno de Navarra.
Placarte, R. (2015). ¿Son racionales las normas sociales? Revista estudios sobre Estado y sociedad, 22(64), pp. 10-40.

Rawls, J. (2003). Teoría de la justicia. México: Fondo de Cultura Económica.

Rivera, C. (2008). El cuidado: Una alternativa en la educación moral. Bogotá: Universidad Javeriana.

Rodríguez, G. (2003). Infuencia del desarrollo moral, las emociones y la identidad sobre la acción moral. Tesis Maestría en Psicología no publicada. Bogotá. Universidad de los Andes.

Rodríguez, G. (2008). Sentimientos y actitudes en la escuela, en educación, valores y ciudadanía. Madrid: OEI.

Rojas, L. (2009). El sentimiento de culpa. Madrid: Aguilar.

Sánchez, J., y Sanz, A. (2011). ¿Qué es una norma social? Una discusión de tres aproximaciones analiticas. Revista internacional de sociología, 69 (3), pp. 661-583.

Sánchez, I. (2009, octubre 8). Clima escolar y resolución de conflictos. Obtenido desde https://inmasanchez.wordpress.com/2009/12/27/ clima-escolar-y-resolucion-de-conflictos/

Secretaría de Educación de Bogotá (SED). (2010). Reorganización curricular por ciclos. Referentes conceptuales y metodológicos. Bogotá: SED.

Secretaría de Educación de Bogotá (SED). (2013). Encuesta de clima escolar y victimización. Bogotá: SED.

Smetana, J. (1997). Crianza y el desarrollo de conocimiento social reconceptualizado: Análisis en el dominio social. En las relaciones y la internalización de los valores para niños. Nueva York.

Villegas de Posada. (2002). Educación para el desarrollo moral. Bogotá: Universidad de los Andes y Alfaomega.

Voli, F. (2004). Sentirse bien en el aula. Manual de convivencia para profesores. Madrid: Educar. 